\title{
Critical Short Circuit Ratio Analysis on DFIG Wind Farm with Vector Power Control and Synchronized Control
}

\author{
Min Hong*, Huanhai Xin*, Weidong Liu**, Qian Xu**, Taiying Zheng ${ }^{\dagger}$ and Deqiang Gan*
}

\begin{abstract}
The introduction of renewable energy sources into the AC grid can change and weaken the strength of the grid, which will in turn affect the stability and robustness of the doubly-fed induction generator (DFIG) wind farm. When integrated with weak grids, the DFIG wind turbine with vector power control often suffers from poor performance and robustness, while the DFIG wind turbine with synchronized control provides better stability. This paper investigates the critical short circuit ratios of DFIG wind turbine with vector power control and synchronized control, to analyze the stability boundary of the DFIG wind turbine. Frequency domain methods based on sensitivity and complementary sensitivity of transfer matrix are used to investigate the stability boundary conditions. The critical capacity of DFIG wind farm with conventional vector power control at a certain point of common coupling (PCC) is obtained and is further increased by employing synchronized control properly. The stability boundary is validated by electromagnetic transient simulation of an offshore wind farm connected to a real regional grid.
\end{abstract}

Keywords: Weak grid, DFIG wind farm, Vector power control, Synchronized control, Stability boundarly, Critical short circuit ratio, Sensitivity

\section{Introduction}

The integration of large numbers of renewable energy sources will change the strength of $\mathrm{AC}$ grid and make the grid weak, which is characterized by low short circuit ratio (SCR) or low inertia [1]. SCR is closely related to voltage stability, so the lower the SCR of a wind farm at the PCC, the more sensitive the voltage fluctuation will be. That will lead to instability and cause wind turbines to trip leading to deterioration in grid stability [2], especially in regions like the Northwest China where there is a large concentration of wind farms connected to a relatively weak power grid $[3,4]$. Hence, with increasing numbers of offshore wind farms under planning and construction in East China, it is necessary to investigate the stability mechanism of wind farms connected to weak grids.

The vector power control is widely adopted in wind farms. The principle of vector power control is to utilize the phase lock loop (PLL) to track the angle of grid voltage or stator flux. The angle produced by PLL is used as the Park transform angle to decouple active power and reactive power control [5]. However, the vector power control is based on the assumption of a strong $\mathrm{AC}$ grid, and its robustness and performance are dependent on the accuracy

$\dagger \quad$ Corresponding Author : College of Electrical Engineering, Zhejiang University, China. (taiying_zheng@zju.edu.cn)

* College of Electrical Engineering, Zhejiang University, China. (\{hongm, xinhh, dgan\}@zju.edu.cn)

** Economic Research Institute of Stage Grid Zhejiang Electric Power Company, China. (liu_weidong@zhejiang.sgcc.com.cn, 596113908@qq.com)

Received: January 8, 2015; Accepted: November 4, 2015 and responsiveness of PLL [6]. When the grid is strong, wind farms with vector power control will enjoy robustness and good performance, and track the power reference quickly and precisely.

When the grid is weakened to a certain extent, the PLL will be unable to detect the violent change of grid angle induced by disturbances timely. Thereby, the error of PLL will make it hard to decouple active power and reactive power control, and even cause instability and tripping of the wind turbine if the disturbance is big enough. In time domain eigenvalue analysis, it is also discovered that the damping of oscillation modes related to PLL is weakened with lower SCR $[7,8]$. In the weak grid with low SCR, the complex dynamics of terminal voltage may destabilize PLL leading to instability within the DFIG system [9]. Consequently, the robustness and performance of the wind turbine are bound up with the SCR of the wind turbine terminal. The robustness and performance of the DFIG wind farm are good with high SCR and poor with low SCR. So it is necessary to study the critical SCR of vector power control under which the stability margin is unacceptable. In [10], the effect of SCR on the static voltage stability is studied. In [11], the effect of SCR on the dynamic performance of wind turbine is investigated through timedomain simulations. The effect of grid strength on the eigenvalues of the wind turbine is investigated in [12]. However, few pieces of research give the exact critical SCR.

Once the inherent weakness of vector power control is known, effective methods can be applied to solve the problem. The two easiest solutions are enhancing the 
voltage stability by Static Synchronous Compensator (STATCOM) [13], [14] and utilizing the free capacity of the wind farm to compensate for the PCC voltage [15]. However, both of the two methods have weaknesses since they are both based on vector power control that will fail under weak grids. As a result of this, synchronized control based on frequency and voltage droop was proposed and has already been applied in the distributed generation [16] of islanded and grid-connected micro-grids $[17,18]$. The synchronized control enjoys good robustness and performance in weak grids [19], but not so good in strong grids [7]. Therefore, it is also necessary to study the critical SCR of the wind farm with synchronized control.

Based on the above considerations, the purpose of this paper is to analyze the critical SCRs of DFIG wind farm with vector power control and with synchronized control respectively. The sensitivity and complementary sensitivity of the transfer matrix in the frequency domain are used to study the quantitative relationship between SCR and DFIG wind farm's robustness and performance. The sensitivity and complementary sensitivity of the wind farm under two control methods are calculated under various SCRs. The critical SCRs are derived from the proposed sensitivity constraints of both the single-input-single-output (SISO) and multi-input-multi-output (MIMO) systems. Finally, the results of frequency domain analysis are validated by electromagnetic transient simulations of an offshore wind farm connected to a real regional power grid in East China.

\section{Modeling and Control of DFIG Wind Farm}

In this section, the dynamic models of the wind farm with vector power control and with synchronized control are established respectively. The wind farm model under study is shown in Fig. 1, where the external grid is simplified as an ideal voltage source. The parameters referred to the model and control are listed in Table 1.

\subsection{Mechanical and electrical model of DFIG}

The wind turbine is modeled as a two-mass module including the torsion between the high-speed shaft and low-speed shaft. The electromagnetic dynamic behavior of DC capacitor, the equivalent grid inductance, and the grid side converter (GSC) inductance are all modeled in detail.

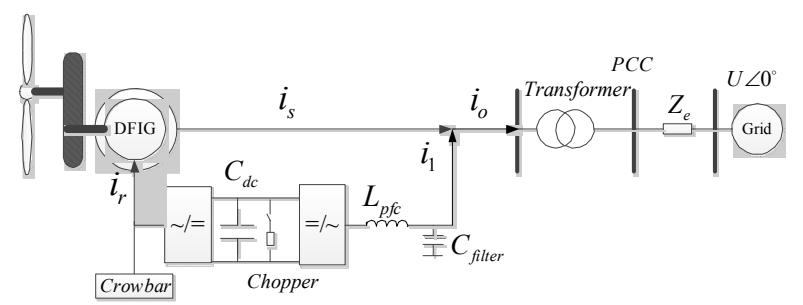

Fig. 1. The topology of the investigated wind farm
Table 1. Main variables in model and control

\begin{tabular}{ll}
\hline \multicolumn{1}{c}{ Variables } & \multicolumn{1}{c}{ Representation } \\
\hline$v_{s d} / v_{s q}, v_{r d} / v_{r q}$ & stator and rotor voltage in dq coordinate \\
$i_{s d} / i_{s q}, i_{r d} / i_{r q}$ & stator voltages and currents in dq coordinate \\
$I_{m s}, I_{m s r e f}$ & excitation current and reference \\
$\psi_{s d} / \psi_{s q}, \psi_{r d} / \psi_{r q}$ stator and rotor fluxes in dq coordinate \\
$P_{s}, Q_{s}, P_{s r e f}, Q_{s r e f}$ & stator active and reactive power, reference \\
$\omega_{e}, \omega_{r}, \omega_{s}$ & stator fluxes speed, rotor speed, slip speed \\
$L_{s}, L_{r}, L_{m}, L_{p f c}$ & stator, rotor, mutual and GSC inductance \\
$C_{d c}, C_{f i l e r}$ & DC capacitor, filter capacitor \\
$L_{e}, R_{e}$ & equivalent inductance and resistance \\
$V_{d c}, V_{d c r e f}$ & DC voltage and reference \\
$i_{g d}, i_{g q}$ & grid side converter current in dq coordinate \\
\hline
\end{tabular}

The DFIG is expressed as the well-known $4^{\text {th }}$-order model under the dq rotating axes:

$$
\begin{gathered}
\left\{\begin{array}{c}
d \psi_{s d} / d t=u_{s d}+\omega_{1} \psi_{s q}-R_{s} i_{s d} \\
d \psi_{s d} / d t=u_{s q}-\omega_{1} \psi_{s d}-R_{s} i_{s q} \\
d \psi_{r d} / d t=u_{r d}+\left(\omega_{1}-\omega_{r}\right) \psi_{r q}-R_{r} i_{r d} \\
d \psi_{r q} / d t=u_{r q}-\left(\omega_{1}-\omega_{r}\right) \psi_{r d}-R_{r} i_{r q}
\end{array}\right. \\
\left\{\begin{array}{l}
\psi_{s r}=L_{s} i_{s r}+L_{m} i_{r r} \\
\psi_{s i}=L_{s} i_{s i}+L_{m} i_{r i} \\
\psi_{r r}=L_{m} i_{s r}+L_{r} i_{r r} \\
\psi_{r i}=L_{m} i_{s i}+L_{r} i_{r i}
\end{array}\right.
\end{gathered}
$$

The rotating speed of dq axes is obtained from PLL in vector power control. In synchronized control, the rotating speed of dq axes is obtained from the active power droop loop.

\subsection{Vector power control of rotor side converter}

The discussed vector power control is based on stator voltage orientation [20]. The target is to track the active power and reactive power references. The active power reference is obtained from the maximum power point tracking (MPPT) curve, which is in proportion to the cubic of the rotor speed. The reactive power reference is generated to keep the power factor of the whole wind farm constant. The decoupled control of active and reactive power is achieved by detecting the grid voltage via PLL. The rotor side converter (RSC) controller consists of the outer stator power loop and inner rotor current loop. The rotor current reference in dq coordinate is generated by the outer power control loop. A typical PLL is applied in the vector power control. The dynamic behavior of PLL is considered in modeling.

\subsection{Synchronized control of rotor side converter}

The synchronized control of the inverter has been widely 


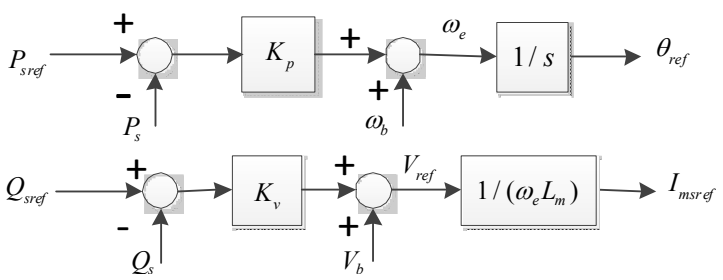

Fig. 2. The outer loop of the synchronized control strategy for DFIG

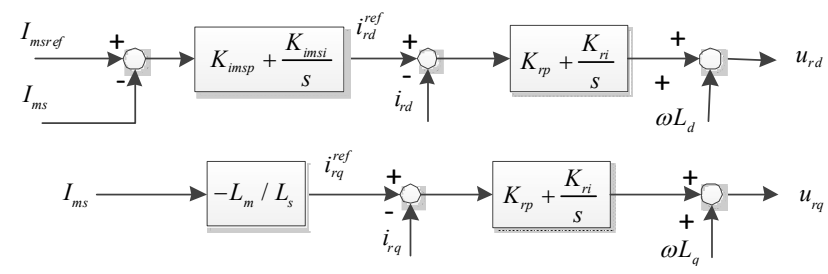

Fig. 3. The excitation and inner rotor current loop of the synchronized control strategy for DFIG

adopted in the micro-grids with high penetrations of renewable resources, where the system is difficult to control. The purpose of synchronized control is to make the nonsynchronous power electronic sources emulate the synchronous generators to provide functions of frequency control, inertial response and voltage regulation. The synchronized control strategy of DFIG consists of the power droop loop, the excitation current outer loop and the rotor current inner loop [21], which is shown in Fig. 2 and Fig. 3. The active power and reactive power references are identical to those of vector power control.

$\omega_{b}$ and $V_{b}$ are usually set at $1 . \omega_{e}$ is the rotating speed of stator flux. $V_{\text {ref }}$ is the reference value of stator voltage.

\subsection{Grid-side converter control}

The grid side converter (GSC) control strategies of the DFIG wind turbines are identical under different RSC control methods. The target of GSC is to make the DC voltage as smooth as possible. The control strategy is shown in Fig. 4. The decoupling of active and reactive power is also fulfilled by voltage orientation.

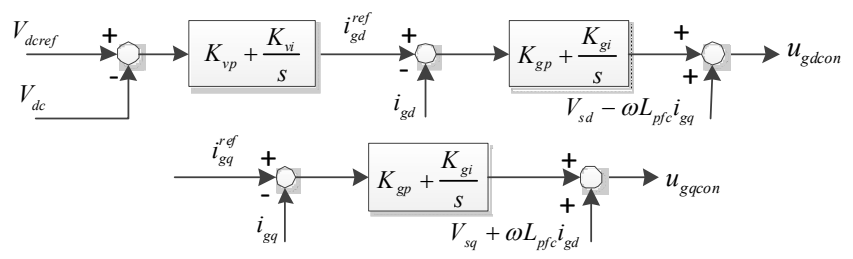

Fig. 4. The GSC control strategy

\section{Frequency-domain Analysis of Wind Farm}

In this section, wind farms with vector power control and synchronized control are both analyzed in the frequency

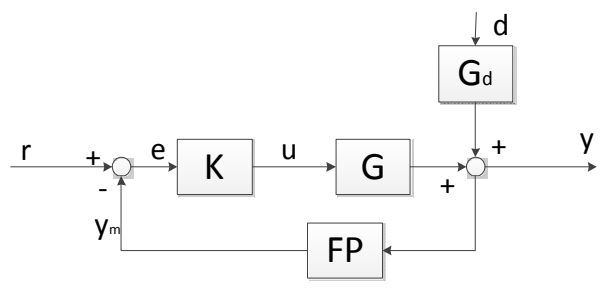

Fig. 5. Common structure of a negative feed-back control system

domain. The wind turbine is regarded as a negative feedback control system. The sensitivity and complementary sensitivity are calculated based on the state space equation.

\subsection{Negative feedback control system and its indexes}

The wind turbine is considered as a one-degree-freedom negative feedback control system as shown in Fig. 5, to analyze the control performance and robustness. $\mathrm{K}$ is the controller while $\mathrm{G}$ is the transfer function of the controlled object. FP is the transfer function from the outputs to feedback signals and is usually designed as a filter.

The response of the close-loop system can be written as below:

$$
y=T * \mathrm{r}+S * \mathrm{G}_{d} * d
$$

where, $\mathrm{T}$ stands for the complementary sensitivity that is the close-loop transfer function from the reference signals to outputs, and S stands for the sensitivity that is the closeloop transfer function from the output disturbances to outputs. S and T can be given as below:

$$
\begin{gathered}
S=\left(I+G^{*} K^{*} F P\right)^{-1} \\
T=\left(I+G^{*} K^{*} F P\right)^{-1} G^{*} K^{*} F P
\end{gathered}
$$

Complementary sensitivity function $\mathrm{T}$ can indicate how well the system tracks the reference while sensitivity function $\mathrm{S}$ represents the capability of tolerating the disturbance. Under the typical condition, $\mathrm{M}_{\mathrm{S}}$ (maximum peak values of $S$ ) should be less than $2(6 \mathrm{db})$, and $M_{T}$ (maximum peak values of $\mathrm{T}$ ) should be less than $1.25(2 \mathrm{db}$ ) [22]. The main difference between a scalar SISO system and an MIMO system is the presence of directions in the latter. The singular value decomposition (SVD) provides a useful way of quantifying multivariable directionality. The sensitivity constrained condition of the SISO system involving the absolute value can be generalized to the MIMO system as below [23]:

$$
\bar{\sigma}(S(j \omega))<2, \forall \omega
$$

where $\bar{\sigma}(S(j \omega))$ is the maximum singular value of the matrix S. It should be noted that the constraints on 
$\bar{\sigma}(T(j \omega))$ (the maximum singular value of Matrix T) are not yet required in any references.

\subsection{Calculation method of $S$ and $T$ for wind turbine}

The first step is to build the small signal dynamic model of the wind farm by linearizing the dynamic model on the equilibrium point. The second step is to select the proper reference signals, outputs and control variables to construct the desired feedback control system. Since the GSC control methods are identical, only the stator active and reactive power are selected as the outputs for convenience:

$$
\left\{\begin{array}{c}
r=\left[P_{\text {sref }}, Q_{s r e f}\right]^{T} \\
y=\left[P_{s}, Q_{s}\right]^{T}
\end{array}\right.
$$

Considering the DFIG wind turbine as a double input and double output system, $\mathrm{S}$ and $\mathrm{T}$ are represented by $2 \times 2$ matrices as shown in (8) and (9). The diagonal elements of $\mathrm{S}$ and $\mathrm{T}$ matrices denote the robustness and performance of active and reactive power control respectively, while the off-diagonal elements denote the coupling between active and reactive power.

$$
\begin{aligned}
& S=\left[\begin{array}{ll}
S_{11} & S_{12} \\
S_{21} & S_{22}
\end{array}\right] \\
& T=\left[\begin{array}{ll}
T_{11} & T_{12} \\
T_{21} & T_{22}
\end{array}\right]
\end{aligned}
$$

By describing the system in the uniform state space equation form of $[A, B, C, D]$, the transfer function of the controlled object $\mathrm{G}$ can be derived as:

$$
G=\left(C(\mathrm{sI}-\mathrm{A})^{-1} B+D\right)
$$

Both of the outer loops of two control strategies are excluded from the state space equation above, since they are independently modeled as the controller $\mathrm{K}$. The wind turbine, DFIG, GSC, electrical interface equations and inner control loops of RSC are all included in the transfer function matrix of the controlled object $\mathrm{G}$.

The third step is to calculate the corresponding sensitivity and complementary sensitivity. The controller K and control variables $\mathrm{u}$ are designed differently in two control strategies.

\subsubsection{Vector power control}

The control variables of vector power control can be defined as:

$$
u=\left[\Delta i_{r d}^{r e f}, \Delta i_{r q}^{r e f}\right]^{T}
$$

The controller $\mathrm{K}$ representing the outer stator power control loop is set as:

$$
\mathrm{K}(\mathrm{s})=\left[\begin{array}{cc}
\frac{K_{s p}}{\left(1+1 /\left(s T_{s p}\right)\right)} & 0 \\
0 & \frac{K_{s q}}{\left(1+1 /\left(s T_{s q}\right)\right)}
\end{array}\right]
$$

The feedback process is usually designed as a first order filter. The sensitivity and complementary sensitivity can be calculated by (4)-(5). $K_{s p}$ and $T_{s p}$ are the proportional coefficients and the integration time constant, respectively.

\subsubsection{Synchronized control}

The control variable $u$ of synchronized control can be defined as:

$$
u=\left[\Delta \omega_{e}, \Delta i_{m s}^{r e f}\right]^{T}
$$

The controller $K$ that represents the outer stator power droop control loop is set as:

$$
\mathrm{K}(\mathrm{s})=\left[\begin{array}{cc}
K_{p} & 0 \\
-\left(K_{p} V_{0} / L_{m} \omega_{e 0}^{2}\right) & \left(K_{q} / L_{m} \omega_{e 0}\right)
\end{array}\right]
$$

where $\omega_{e 0}$ is the initial value of the frequency $\omega_{e}$.

\section{Stability Boundaries Analysis}

In this section, the stability boundary conditions of the DFIG wind turbine on SCR are derived from the frequency analysis method introduced in the previous section.

$\mathrm{S}$ and $\mathrm{T}$ values are represented in the frequency range from $10^{-2} \mathrm{rad} / \mathrm{s}$ to $10^{7} \mathrm{rad} / \mathrm{s}$. In terms of $\mathrm{S}$ and $\mathrm{T}$ analysis, the whole frequency band can be divided into four parts:

1) ultralow frequency band (from $10^{-2} \mathrm{rad} / \mathrm{s}$ to $10^{0} \mathrm{rad} / \mathrm{s}$ );

2) low frequency band (from $10^{0} \mathrm{rad} / \mathrm{s}$ to $10^{1} \mathrm{rad} / \mathrm{s}$ );

3) middle frequency band (from $10^{1} \mathrm{rad} / \mathrm{s}$ to $10^{2} \mathrm{rad} / \mathrm{s}$ );

4) high frequency band (above $10^{2} \mathrm{rad} / \mathrm{s}$ ).

Considering the DFIG wind turbine as a double-input and double-output system, the stability boundary conditions can be defined as below:

1) $\bar{\sigma}(S(j \omega))$ should be smaller than 2 in the whole frequency scope as mentioned in (6);

2) $T_{11}$ and $T_{21}$ should be less than $1.25(2 \mathrm{~dB})$ around the frequency of $1.5 \mathrm{~Hz}$ to prevent low frequency oscillation stimulated by the wind turbine shaft;

3 ) the whole elements of the Matrix S should be smaller than $0 \mathrm{~dB}$ within the ultralow frequency band in order to ensure robustness and stability. 
Elements of matrices $\mathrm{S}$ and $\mathrm{T}$ are represented in $\mathrm{dB}$ form. To obtain the stability boundary conditions on SCR, the sensitivity and the complementary sensitivity need to be calculated under various SCRs for both control methods. Only the full load condition is analyzed in this section because it can get the stability boundary condition. In this case, the rotor speed is $1800 \mathrm{rpm}$ (1.2.pu), and the active power output is about $100 \%$ of rated power under all SCRs. The reactive power output varies according to different SCRs to keep the stator terminal voltage within the acceptable scope.

In the frequency-domain analysis, the power system is simplified as an infinite bus with an equivalent system impedance. A real regional power grid is adopted in the time-domain simulations to further validate the stability boundaries derived from the frequency-domain analysis.

\subsection{Stability boundaries of vector power control}

The stability boundary condition can be derived from the maximum singular value of sensitivity under this situation. The curves for maximum singular values of sensitivity are shown in Fig. 6.

\subsubsection{Ultralow frequency band}

The maximum singular values of sensitivity are about 0 and the maximum singular values of complementary sensitivity are about 1 under all SCRs in this band. It is indicated that the performance and robustness of active power control for vector power control are preferable in the ultralow-frequency band (below 1rad/s) under all the SCRs.

\subsubsection{Low frequency band}

The maximum singular values of sensitivity begin to rise, while the maximum singular values of complementary sensitivity begin to fall in this band which means the robustness of active power control in this range is weakened, but still acceptable. Although the natural oscillation frequency of the wind turbine shaft falls into

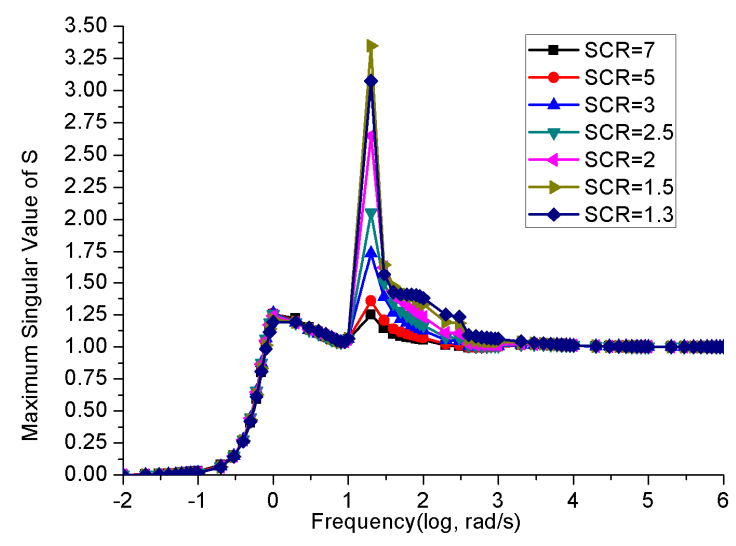

Fig. 6. Maximum singular value of sensitivity for the vector power control this band, the values of $T_{11}$ keep close to $0 \mathrm{~dB}$, which indicates a good capability of tracking the active power reference within this band.

\subsubsection{Middle frequency band}

In this band, the values of $\bar{\sigma}(S(j \omega))$ begin to diverge obviously with different SCRs. $\bar{\sigma}(S(j \omega))$ and $\bar{\sigma}(T(j \omega))$ all reach their peak values, and the peak values increase almost monotonically with the decreasing SCR. When the SCR gets lower than 2.5, the peak value of $\bar{\sigma}(S(j \omega))$ gets higher than 2. It's indicated that the performance and robustness of active power control in this frequency scope get worse with the decreased SCR. According to the first item of stability criteria conditions proposed above, the SCR should be larger than 2.5. The high sensitivity under small SCR reveals that the DFIG wind turbine may cause sub-synchronized oscillation of the nearby synchronous generators. It is already known that sub-synchronized resonance may happen in DFIG wind farms connected to the series-compensated transmission line [24].

\subsubsection{High frequency band}

From $10^{2} \mathrm{rad} / \mathrm{s}$ to $10^{3} \mathrm{rad} / \mathrm{s}$, it can be observed that the values of $\bar{\sigma}(S(j \omega))$ and $\bar{\sigma}(T(j \omega))$ still increase with the decreased SCR because of the off-diagonal elements of $\mathrm{S}$ and $\mathrm{T}$ matrices. That means the coupling of active and reactive power within this frequency range gets stronger with decreased SCR. But with increasing frequency, all the values of $\bar{\sigma}(S(j \omega))$ approach 1 despite different SCRs. That means the active power disturbance at the high frequency gets neither larger nor smaller.

Based on the analysis above, it can be found that the performance and robustness of vector power control get worse with decreased SCR. Therefore, the stability boundary condition on SCR is 2.5 in this situation.

\subsection{Stability boundaries of synchronized control}

It is from the values of $\mathrm{S}_{22}$ that the stability boundary

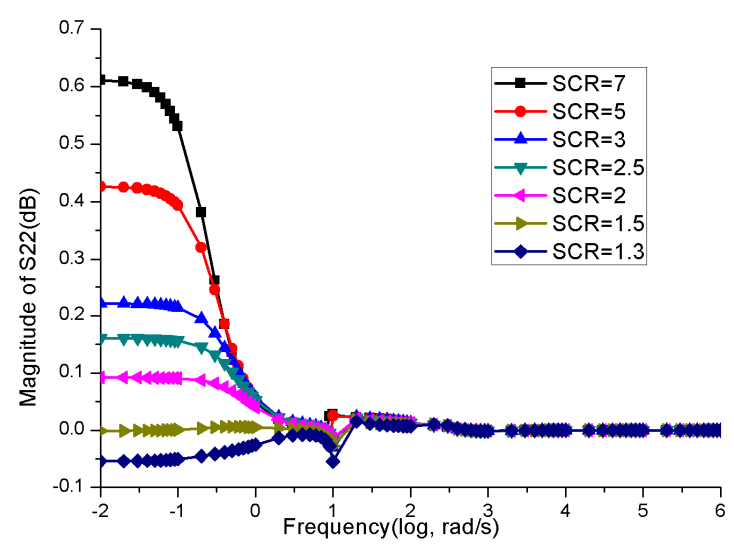

Fig. 7. $S_{22}$ for the synchronized control 


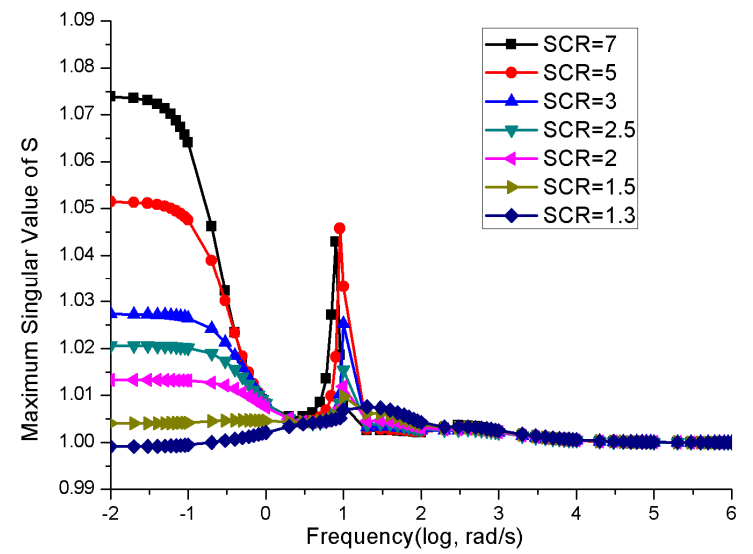

Fig. 8. Maximum singular value of sensitivity for the synchronized control

condition can be derived under this situation. So the values of $S_{22}$ and the maximum singular values of sensitivity are plotted in Fig. 7 and Fig. 8 respectively. The former one is in the $\mathrm{dB}$ form and the latter one is in the normal form.

\subsubsection{Ultralow frequency band}

In this frequency band, the curves and trends of $S_{11}$, $S_{12}, T_{11}$ and $T_{12}$ are not obviously different from those of vector power control, which means the performance and robustness of active power under synchronized control differ little with those under vector power control within the band. However, for reactive power control, the value of $S_{22}$ does not become lower than 0dB until the SCR decreases to 1.5 . The fact that $T_{22}$ within this band becomes lower than $0 \mathrm{~dB}$ means that the reactive power reference within this frequency band is unable to be tracked accurately and quickly. Meanwhile, the oscillation within this frequency band is hard to be mitigated. That can be explained by the reason that the reactive power control is based on reactive power droop without an integral loop. That will cause the transient over-voltage to last comparatively longer after the clearance of grid fault. According to the second item of stability criteria proposed above, the SCR should be smaller than 1.5.

\subsubsection{Low frequency band}

Within this band, values of $S_{11}, S_{12}$ and $T_{12}$ are still below $-20 \mathrm{~dB}$ and values of $T_{11}$ are close to $0 \mathrm{~dB}$, so the active power is still controllable under various SCRs. Values of $S_{22}$ with SCRs more than 1.5 have all decreased to $0 \mathrm{~dB}$. The off-diagonal elements and maximum singular values of $\mathrm{S}$ and $\mathrm{T}$ matrices all reach their peak values at the frequency of $10^{1} \mathrm{rad} / \mathrm{s}$ but are far below limit values defined by stability boundaries above. That means the coupling of active power and reactive power around $10^{1}$ $\mathrm{rad} / \mathrm{s}$ is the most severe but still acceptable.

\subsubsection{Middle frequency band}

Within this band, the elements of Matrix S are too low to be discussed. Meanwhile, the maximum singular values of Matrix S are far lower than 2. Therefore, the robustness of reactive power control for synchronized control within this band is much better than that of vector power control.

\subsubsection{High frequency band}

The diagonal elements of Matrix $\mathrm{S}$ all tend to $0 \mathrm{~dB}$, while the off-diagonal elements of Matrix $\mathrm{S}$ and the whole elements of Matrix T all decrease sharply as the frequency increases. It is quite different from the vector power control.

As for active power control, the performance and robustness of synchronized control are much better than those of vector power control under SCR smaller than 5 . While for reactive power control, the performance and robustness of synchronized control are worse than those of vector power control in the ultralow frequency band. But they are better in other frequency bands, especially when SCR is smaller than 2.5. Based on the analysis above, the critical SCR of DFIG with synchronized control is 1.5 .

However, the stability boundary condition of synchronized control is comparatively vaguer than that of vector power control, because the differences of $S_{11}$ among different SCRs in ultralow frequency band are not so obvious. It can be concluded that the performance and robustness of synchronized control are better under weak grids, but worse under strong grids, compared with vector power control

\section{Case Study}

The critical SCRs are validated by simulations of an offshore wind farm connected to a real regional power grid in East China as shown in Fig. 9.

The electromagnetic transient behavior of the whole grid including wind farms is considered in the simulation model. The regional grid consists of 37 nodes with voltage levels at and above $220 \mathrm{kV}$. Before the integration of wind farm, the total generation power of the regional grid is $3310 \mathrm{MW}$, and the total load is $2457 \mathrm{MW}$. The external grid is simplified as an equivalent generator shown in the red circle. There are three thermal plants consisting of five generators in this regional grid. All the synchronous generators are expressed by the classic $6^{\text {th }}$ order model with the detailed excitation controller, power system stabilizer, and turbine governor. The shafts of synchronous generators are modeled as 6mass modules. $60 \%$ of the loads are set as constant power loads, and the rests are set as constant impedance loads. The simulations are carried out under the full-load condition. The rotor speed is $1800 \mathrm{rpm}$, and the total active power output at PCC is about $100 \%$ of rated power before the grid fault. The simulations are executed via commercial 


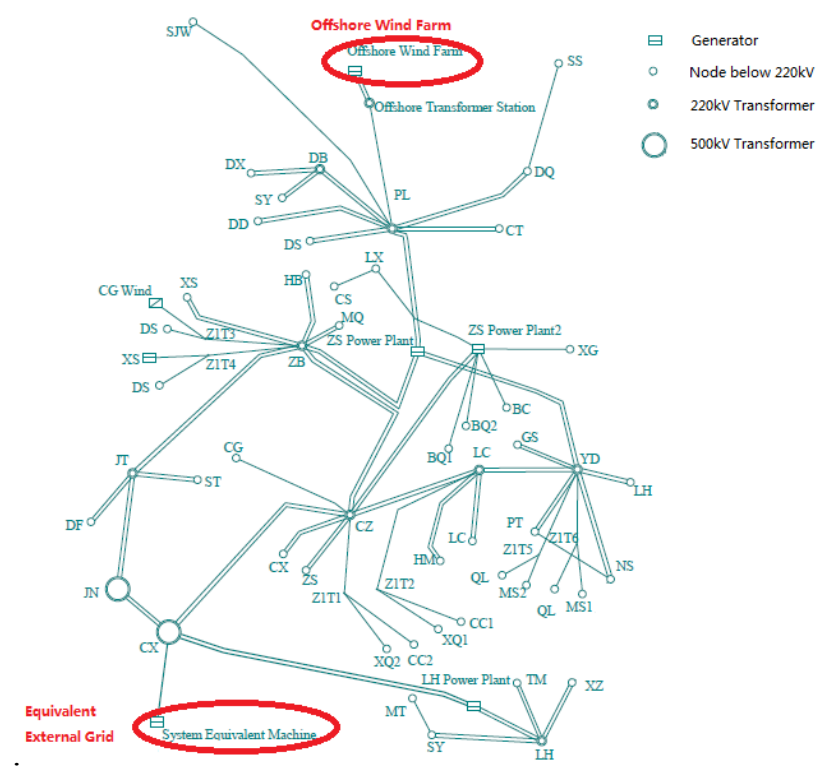

Fig. 9. The geographic graph of the regional power grid in East China connected with the offshore wind farm

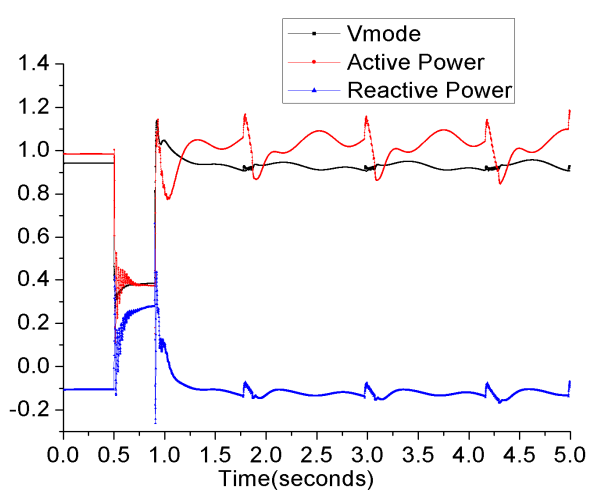

Fig. 10. Response of DFIG with vector power control to a three-phase voltage dip $(\mathrm{SCR}=2.5)$

simulation software DIgSILENT.

\subsection{Case A}

The capacity of the wind farm is $600 \mathrm{MW}$, and the SCR at the wind turbine terminal is 2.5. Vector power control is applied to the DFIG wind farm. A three-phase voltage dip to $20 \%$ is imposed on the $220 \mathrm{kV}$ bus-bar of the offshore booster station and cleared after $400 \mathrm{~ms}$. The simulation results are shown in Fig. 10. It can be found that after fault clearance, the system is unable to return to the equilibrium point before the fault. The stator voltage of the wind turbine frequently falls below $90 \%$. According to the common protection setting and grid code [25], that will lead to the frequent triggering of low voltage ride through (LVRT) signal. The wind turbine frequently switches between normal mode and LVRT mode, which gives rise to the fluctuation of the active and reactive power. Consequently, the wind turbine will trip due to the protection scheme. It

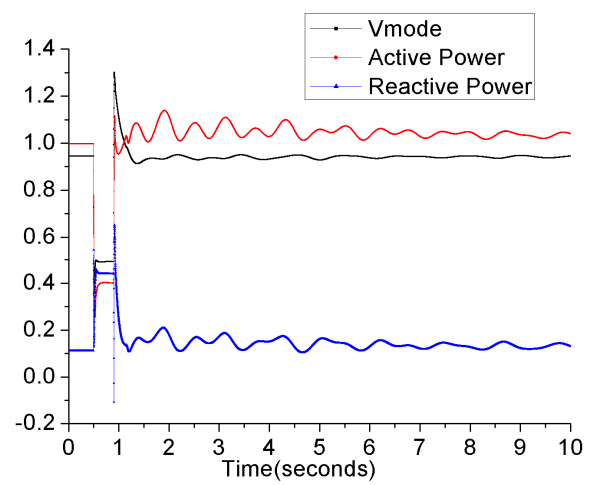

Fig. 11. Response of DFIG with synchronized control to a three-phase voltage dip $(\mathrm{SCR}=1.5)$

can be validated critical SCR of DFIG with vector power control is about 2.5 .

\subsection{Case B}

The capacity of the wind farm is $900 \mathrm{MW}$, and the SCR at the wind turbine terminal is 1.5 . Synchronized control is applied to the DFIG wind farm. A three-phase voltage dip to $30 \%$ is imposed on the $220 \mathrm{kV}$ bus-bar of the offshore booster station and cleared after $400 \mathrm{~ms}$. The simulation results are shown in Fig. 11. It can be discovered that the wind farm remains stable after fault clearance even with SCR smaller than 1.5. That is due to the reactive power droop loop of synchronized control, which guarantees better voltage stability under the weak grid since the wind turbine works as a voltage source. Meanwhile, the synchronized control can produce higher residual voltage with the same amount of reactive current compared with the vector power control.

The only problem is the temporary transient over voltage immediately after the fault clearance. These phenomena can all be explained by the fact that the DFIG wind turbine with synchronized control works as a voltage source. However, this can also be solved by the optimization of the fault ride through control strategy of the synchronized control.

\subsection{Case C}

The wind farm capacity, SCR and voltage dip are the same with those of case B. Vector power control is applied to the DFIG wind farm. The simulation results are shown in Fig. 12. It can be found that after fault clearance, the grid voltage is unable to be restored to $90 \%$ of the nominal level. During the transient process after the grid fault clearance, there are big fluctuations of active and reactive power. After the transient process, the active power slowly rises, and voltage gradually falls. Finally, the system moves to the saddle point of the PV curve, and the voltage collapses occur 9 seconds after the fault clearance. It can be validated that the DFIG wind turbine with vector power 


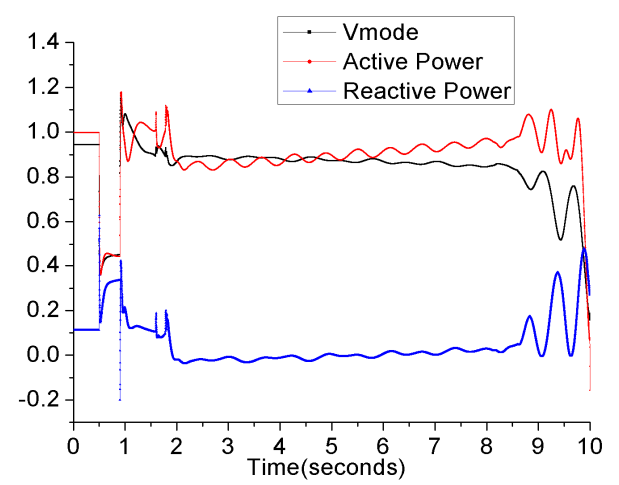

Fig. 12. Response of DFIG with vector power control to a three-phase voltage dip $(\mathrm{SCR}=1.5)$

control is not stable in weak grids with SCR smaller than 1.5.

\section{Conclusions}

In this paper, the critical SCRs of DFIG wind turbine with vector power and synchronized control are derived from frequency-domain analysis respectively. Time-domain electromagnetic transient simulations are performed to validate the results, where an offshore wind farm is connected to a real regional grid. It is shown that the vector power control is more suitable for strong grids while the synchronized control is more suitable for weak grids. With the critical SCR at hand, the maximum acceptable capacity of wind power at a certain bus can be obtained when the control method is known. Moreover, the control mode can be adapted according to the SCR, i.e., the vector power control can be used when the grid is strong or synchronized control if adopted grid strength falls. An adaptive control strategy combining the advantages of the two control methods will be proposed in our future work.

\section{Acknowledgements}

This work is jointly supported by the project of the State Grid (No. No.B311JY13001Z and No.JY02201407), the National Science Foundation of China (No.51177146) and the Department of Science \& Technology program (No.2011AA05A105).

\section{References}

[1] "IEEE Guide for Planning DC Links Terminating at AC Locations Having Low Short-Circuit Capacities," IEEE Std 1204-1997, Tech. Rep., 1997.

[2] T. Neumann, C. Feltes, and I. Erlich, "Response of DFG-based wind farms operating on weak grids to voltage sags," in Proc. IEEE Power Eng. Soc. General Meeting, 2011, pp. 1-6.
[3] X. Xing-wei, M. Gang, S. Guang-hui, Z. Hong-peng, H. Kai-yuan, G. De-bin, T. Jia-qi, M. Xin, and X. Yong, "The problems and solutions for large-scale concentrated integration of wind power to partially weak regional power grid," in Proc. SUPERGEN, 2009, pp. 1-6.

[4] D. Peng, Z. Yiying, L. Chong, H. Tao, and W. Weiwei, "The impact of LVRT characteristic on the stability of northwest China grid with large scale wind power," presented at the Renewable Power Generation Conference (RPG 2013), Beijing, 2013.

[5] L. Chen and Z. Li, "The comparison of common control strategies in grid-connected double-fed wind generators," in Proc. TENCON 2013-2013 IEEE Region 10 Conference, 2013, pp. 1-5.

[6] D. Jovcic, L. Lamont, and L. Xu, "VSC transmission model for analytical studies," in Proc. IEEE Power Eng. Soc. General Meeting, 2003, pp. 1737-1742.

[7] M. Hong, H. Xin, Q. Xu, L. Sun, T. Zhen, and D. Gan, "Stability Boundary Investigation of Large-scale offshore DFIG wind Farm Connected to Weak grid," in Proc. Wind energy Grid-Adaptive Technologies, 2014, pp. 1-8.

[8] W. Dong, H. Jiabing, H. Yunhui, W. Ningbo, and Z. Qiang, "Stability of DC-link voltage affected by phase-locked loop for DFIG-based wind turbine connected to a weak AC system," in Proc. ICEMS, 2014, pp. 2600-2606.

[9] Q. Hu, J. Hu, H. Yuan, H. Tang, and Y. Li, "Synchronizing stability of DFIG-based wind turbines attached to weak AC grid," in Proc. ICEMS, 2014, pp. 26182624.

[10] S. Barghi, M. A. Golkar, and A. Hajizadeh, "Effect of distribution system specifications on voltage stability in presence of wind distributed generation," in Proc. EPDC, 2011, pp. 1-6.

[11] L. Wang, C.-J. Yeh, M.-H. Hsieh, C.-T. Wu, and C.-L. $\mathrm{Lu}$, "Analysis of voltage variations and short-circuit ratios of a large-scale offshore wind farm connected to a practical power system," in Proc. IEEE Power Eng. Soc. General Meeting, 2013, pp. 1-5.

[12] N. P. Strachan and D. Jovcic, "Stability of a variablespeed permanent magnet wind generator with weak AC grids," IEEE Trans.Power Delivery, vol. 25, no. 4, pp. 2779-2788, 2010.

[13] K. Jihun., L. Hwanik., L. Byongjun., and K. YongCheol, "Hybrid Secondary Voltage Control combined with Large-Scale Wind Farms and Synchronous Generators," Journal of Electrical Engineering \& Technology, vol. 10, no. 2, pp. 504-510, 2014.

[14] C. Han, A. Q. Huang, M. E. Baran, S. Bhattacharya, W. Litzenberger, L. Anderson, A. L. Johnson, and A.A. Edris, "STATCOM impact study on the integration of a large wind farm into a weak loop power system," IEEE Trans. Energy conversion, vol. 23, no. 1, pp. 226-233, 2008. 
[15] J. Kim, G. Park, J.-K. Seok, B. Lee, and Y. C. Kang, "Hierarchical Voltage Control of a Wind Power Plant Using the Adaptive IQ-V Characteristic of a DoublyFed Induction Generator," Journal of Electrical Engineering \& Technology, vol.10, no.2, pp.504-510, 2015.

[16] K. De Brabandere, B. Bolsens, J. Van den Keybus, A. Woyte, J. Driesen, and R. Belmans, "A voltage and frequency droop control method for parallel inverters," IEEE Trans.Power Electronics, vol. 22, no. 4, pp. 1107-1115, 2007.

[17] Z. Wang, Y. Sun, G. Li, and B. T. Ooi, "Magnitude and frequency control of grid-connected doubly fed induction generator based on synchronised model for wind power generation," IET Renewable Power Generation, vol. 4, no. 3, pp. 232-241, 2010.

[18] M. Fazeli, G. M. Asher, C. Klumpner, and L. Yao, "Novel integration of DFIG-based wind generators within microgrids," IEEE Trans.Energy Conversion, vol. 26, no. 3, pp. 840-850, 2011.

[19] L. Zhang, "Modeling and Control of VSC-HVDC Links Connected to Weak AC Systems," Ph.d, Royal Institute of Technology, Stockholm, 2010.

[20] R. Cardenas, R. Pena, S. Alepuz, and G. Asher, "Overview of Control Systems for the Operation of DFIGs in Wind Energy Applications," IEEE Trans. Industrial Electronics, vol.60, no.7, pp.2776-2798, 2013.

[21] L. Zehan, W. Zhen, X. Huanhai, and W. Kitpo, "Small signal stability analysis of a synchronized controlbased microgrid under multiple operating conditions," Journal of Modern Power Systems and Clean Energy, vol. 2, no. 3, pp. 244-255, 2014.

[22] I. Kamwa, G. Trudel, and L. Gerin-Lajoie, "Robust design and coordination of multiple damping controllers using nonlinear constrained optimization," IEEE Trans.Power Systems, vol. 15, no. 3, pp. 10841092, 2000.

[23] S. Skogestad and I. Postlethwaite, Multivariable feedback control: analysis and design. New York: JOHN WILEY\&SONS, 2007, pp. 40-43.

[24] R. Varma and A. Moharana, "SSR in double-cage induction generator-based wind farm connected to series-compensated transmission line," IEEE Trans. Power Systems, vol. 28, no. 3, pp. 2573-2583, 2013.

[25] E.ON, "Grid Code High and extra high voltage," in Requirements on generating plants, ed. Bayreuth: E.ON Nets GmbH, 2006.

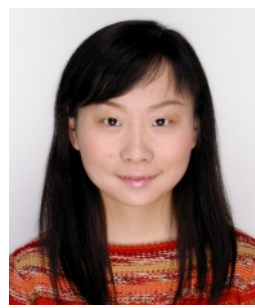

Min Hong She received her B.S and M.S. degree from the Sichuan University, China. She had been working as an electric engineer in the wind power manufacturing company from 2007 to 2012 . She is currently working towards the Ph.D. degree in the College of Electrical Engineering, Zhejiang University, China. Her research interests include renewable energy and power system stability analysis.

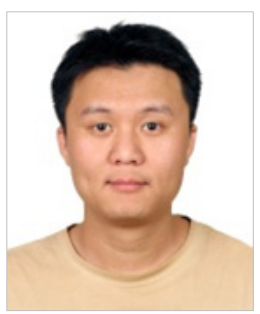

Huan-Hai Xin He received the Ph.D. degree from the Zhejiang University, China. He has been with the faculty, currently a professor in the College of Electrical Engineering, China. His research interests include power system stability analysis, and renewable energy.

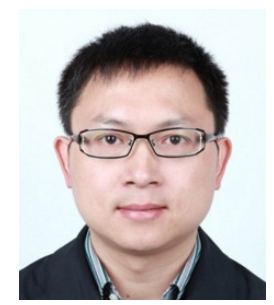

Wei-Dong Liu He is now working in the Economic Research Institute of State Grid Zhejiang Electric Power Company. $\mathrm{He}$ is also purchasing his Ph.D. degree in Xi'an Jiaotong University. His research interests include power system plan.

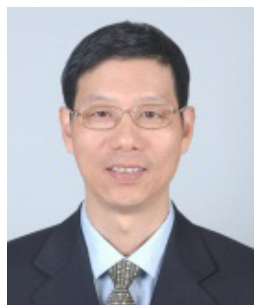

Qian Xu He received B.S degree from the Zhejiang University, China. He is now working as vice chief engineer in the Economic Research Institute of State Grid Zhejiang Electric Power Company. His research interests include power system plan.

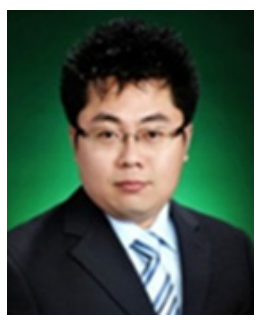

Tai-ying Zheng He received the Ph.D. degree from the Chonbuk National University, Korea. He is currently a lecturer in the College of Electrical Engineering, Zhejiang University, China His research interests include the development of protection systems for power systems using digital signal processing techniques, and the development of new protection / control systems for renewable sources.

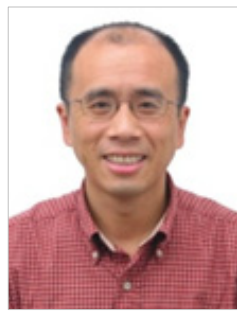

De-qiang Gan He received the Ph.D. degree from the Xi'an Jiaotong University, China. He is a professor in the College of Electrical Engineering, Zhejiang University Zhejiang University, Hangzhou, China, since 2002. His employment experience includes ISO New England, Inc., Ibaraki University, University of Central Florida, and Cornell University. His research interests include power system stability and market operations. 